\title{
THE $a$-POINTS OF FABER POLYNOMIALS FOR A SPECIAL FUNCTION $\dagger$
}

\author{
by HASSOON S. AL-AMIRI \\ (Received 25 May, 1968)
}

1. Introduction. Let $f(\zeta)$ be a power series of the form

$$
\zeta+a_{0}+a_{1} / \zeta+\ldots,
$$

where lim sup $\left|a_{n}\right|^{1 / n}<\infty$. The Faber polynomials $\left\{f_{n}(\zeta)\right\}(n=0,1,2, \ldots)$ are the polynomial parts of the formal expansion of $(f(\zeta))^{n}$ about $\zeta=\infty$. Series (1) defines an analytic element of an analytic function which we designate as $w=f(\zeta)$. Since $f^{\prime}(\zeta) \neq 0$ at $\zeta=\infty$, the analytic element is univalent in some neighborhood of infinity; thus the inverse of this element is uniquely determined in some neighborhood of $w=\infty$, and has a Laurent expansion of the form

$$
w+b_{0}+b_{1} / w+\ldots,
$$

where $\lim \sup \left|b_{n}\right|^{1 / n}=\rho<\infty$. Let $\zeta=g(w)$ be this single-valued function defined by (2) in $|w|>\rho$. No analytic continuation of $g(w)$ will be considered.

Let $\Delta(\zeta)$ and $\Delta_{a}(\zeta)(a \neq 0)$ be the derived sets, in the $\zeta$-plane, of the zeros of $f_{n}(\zeta)$ and $f_{n}(\zeta)-a$, respectively. These sets can be described by means of certain sets in the $\zeta$-plane whose definitions follow:

DEFINITION. $A$ point $\zeta_{1}$, in the $\zeta$-plane, is said to belong to the set $c_{1}$ if $g(w)-\zeta_{1}=0$ has a solution $w_{1}$ in $|w|>\rho$ such that $g^{\prime}\left(w_{1}\right) \neq 0, g\left(w_{2}\right) \neq \zeta_{1}$ for $\left|w_{2}\right| \geqq\left|w_{1}\right|, w_{2} \neq w_{1}$. A point $\zeta_{1}$, in the $\zeta$-plane, is said to belong to the set $s_{1}$ if $\zeta_{1}$ is in $c_{1}$ and the corresponding solution, of greatest modulus, for $g(w)-\zeta_{1}=0$ is of modulus greater than 1 .

Ullman [4] proved the following theorem concerning $\Delta(\zeta)$.

THEOREM 1. (a) $\Delta(\zeta)$ lies in the complement of $c_{1}$ and $(b) \Delta(\zeta)$ contains every boundary point of $c_{1}$.

In [1] the author extended Ullman's results to $\Delta_{a}(\zeta)$ :

THEOREM 2. (a) $\Delta_{a}(\zeta)$ lies in the complement of $s_{1}$ and $(b) \Delta_{a}(\zeta)$ contains every boundary point of $s_{1}$.

Theorem 2 indicates an interesting difference between the cases $\rho>1$ and $\rho<1$. It shows that $a=0$ is a special case when $\rho>1$, while it is an exceptional case when $\rho<1$.

The object of this paper is the location of $\Delta(\zeta)$ and $\Delta_{a}(\zeta)$ for a special function, namely

$$
w=f(\zeta)=\zeta e^{1 /(\lambda \zeta)}=\zeta+1 / \lambda+1 /\left(2 \dot{\lambda}^{2} \zeta\right)+\ldots,
$$

where $\lambda$ is an arbitrary positive number. In $\S 3$ the following theorem concerning the location of $\Delta(\zeta)$ and $\Delta_{a}(\zeta)$ is established. We state the theorem relative to the $z$-plane, where $z=1 / \lambda \zeta$.

† An abstract of this paper was submitted to the seventy-fourth annual meeting of the American Mathematical Society and presented in January, 1968. 
THeOREM 3. (a) $\Delta(z)$ is the set $\Gamma=\left\{z|| z e^{1-z}|=1| z \mid, \leqq 1\right\}$. (b) For $\lambda<e, \Delta_{a}(z)$ is the set $\Gamma$ as in part $(a)$, while for $\lambda \geqq e$ it is the set $\Gamma_{1}=\left\{z|| \lambda z e^{-z}|=1| z \mid, \leqq 1\right\}$.

Finally, in $\$ 4$ an asymptotic distribution of the $a$-points along $\Gamma$ and $\Gamma_{1}$ is established.

2. Discussion of results. The methods used in proving Theorems 1 and 2 are hard to apply for the special function (3). Instead we employ methods used by Szegö [3], which lend themselves naturally to this case.

To obtain the exterior mapping radius $\rho$ associated with (3), we use Bürmann-Lagrange series (see for example [2]) and get

$$
g(w)=w-\sum_{0}^{\infty} \frac{n^{n} w^{-n}}{(n+1) ! \lambda^{n+1}} .
$$

Thus

$$
\rho=e / \lambda
$$

In order to determine the sets $c_{1}$ and $s_{1}$ for the special function, we need to discuss the mapping

$$
\tau=z e^{1-z}
$$

The level curve $\left|z e^{1-z}\right|=1$ is symmetrical with respect to the $x$-axis and consists of two parts:

$$
\left.\begin{array}{rl}
\Gamma & =\left\{z|| z e^{1-z}|=1,| z \mid \leqq 1\right\}, \\
\Gamma^{\prime} & =\left\{z|| z e^{1-z}|=1,| z \mid \geqq 1\right\} .
\end{array}\right\}
$$

From the polar equations of (5), one can easily see that $\Gamma$ is a simple closed curve intersecting the $x$-axis at -0.278 and 1 . The second part $\Gamma^{\prime}$ intersects the $x$-axis at 1 alone; thus the level curve has a double point at $z=1$ and makes the angles $\pi / 4,3 \pi / 4,5 \pi / 4,7 \pi / 4$ with the $x$-axis there. Let I, II and III be the domains interior of $\Gamma$, to the right of $\Gamma^{\prime}$ and bounded by $\Gamma$ and $\Gamma^{\prime}$, respectively. Using the polar equations of (5), one can easily show that (5) maps $I$ in a one-to-one manner onto $|\tau|<1$, and maps II in a similar manner onto the infinite Riemann surface which has been constructed with a cut along the negative $x$-axis, for which $|\tau|<1$. Domain III is mapped by (5) in a similar manner onto the above Riemann surface for which $|\tau|>1$.

Since no analytic continuation is considered for $\zeta=g(w)$, the inverse of the special function, the set $c_{1}$ is easily seen through the transformations $\zeta=1 /(\lambda z), \tau=e /(\lambda w)$ as the set I in the $z$-plane. Similarly $s_{1}$ becomes, in the $z$-plane, the part of I corresponding to $|w| \geqq 1$ or $|\tau| \leqq e / \lambda$. Hence $s_{1}$ is the interior of $\Gamma_{1}$ (See (9) below). Thus to establish Theorems 1 and 2 for the special function (3) is equivalent to proving Theorem 3.

3. Location of $\Delta(z)$ and $\Delta_{a}(z)$. Let

$$
s_{n}(z)=\sum_{0}^{n}(n z)^{p} / p !(n=1,2, \ldots) \text { and } g_{n}(z)=1-e^{-n z} s_{n}(z)
$$


Szegö used the following lemma to show, among other things, that the derived set of the zeros of $s_{n}(z)$ is the curve $\Gamma$ given by (6).

Lemma 1. For $z \neq 1$,

(a) $g_{n}(z)=(1 / \sqrt{ }(2 \pi n))\left(z e^{1-z}\right)^{n}(z /(1-z))\left(1+\varepsilon_{n}(z)\right)$ for $z$ in I, Ill or on $\Gamma$.

(b) $g_{n}(z)=1+(1 / \sqrt{ }(2 \pi n))\left(z e^{1-z}\right)^{n}(z /(1-z))\left(1+\varepsilon_{n}^{\prime}(z)\right)$ for $z$ in II, III or on $\Gamma^{\prime}$.

In $(a)$ and $(b) \lim \varepsilon_{n}(z)=\lim \varepsilon_{n}^{\prime}(z)=0$ uniformly in every finite region which is located entirely in the corresponding regions of $(a)$ and $(b)$ and does not include $z=1$.

Since $\zeta^{n} e^{n / \lambda \zeta}=\zeta^{n}\left(1+n / \lambda \zeta+\ldots+n^{n} / n ! \lambda^{n} \zeta^{n}+\ldots\right)$, the Faber polynomials associated with (3) are given by

From $\zeta=1 /(\lambda z)$,

$$
f_{n}(\zeta)=\zeta^{n}\left\{1+n /(\lambda \zeta)+\ldots+n^{n} /\left(n ! \lambda^{n} \zeta^{n}\right)\right\}
$$

$$
f_{n}(\zeta)=f_{n}(1 / \lambda z)=\left(1+n z+n^{2} z^{2} / 2 !+\ldots+n^{n} z^{n} / n !\right) / \lambda^{n} z^{n}=s_{n}(z) / \lambda^{n} z^{n}
$$

Thus the zeros of $f_{n}(\zeta)$ in the $\zeta$-plane are those of $s_{n}(z)$ in the $z$-plane. It follows then that $\Delta(z)$ is the curve $\Gamma$, which is part $(a)$ of Theorem 3 .

Let $q_{n}(z)=f_{n}(\zeta)-a=s_{n}(z) / \lambda^{n} z^{n}-a$. Substitution yields

$$
g_{n}(z)=1-e^{-n z} s_{n}(z)=1-e^{-n z} \lambda^{n} z^{n}\left(a+q_{n}(z)\right) .
$$

It is now clear that $\Delta_{a}(z)$ is the derived set of the solutions of $g_{n}(z)=1-a e^{-n z} \lambda^{n} z^{n}$. Set

$$
G_{n}(z)=g_{n}(z)+a e^{-n z} \lambda^{n} z^{n}
$$

The set $\Delta_{a}(z)$ becomes the derived set of the solutions of $G_{n}(z)=1$. We need the following lemma in order to locate $\Delta_{a}(z)$ when $\rho=e / \lambda>1$ (See (4)).

LEMMA 2. For $\rho=e / \lambda>1, z \neq 1$ we have

(a) $G_{n}(z)=(1 / \sqrt{ }(2 \pi n))\left(z e^{1-z}\right)^{n}(z /(1-z))\left(1+E_{n}(z)\right)$,

for $z$ in $\mathrm{I}$, III or on $\Gamma$.

(b) $G_{n}(z)=1+(1 / \sqrt{ }(2 \pi n))\left(z e^{1-z}\right)^{n}(z /(1-z))\left(1+E_{n}^{\prime}(z)\right)$,

for $z$ in II, III or on $\Gamma^{\prime}$.

$E_{n}(z)$ and $E_{n}^{\prime}(z)$ have the same limit behavior as $\varepsilon_{n}(z), \varepsilon_{n}^{\prime}(z)$ in Lemma 1 .

The above lemma can be proved easily from Lemma 1. In fact Lemma 2 gives the same representations for $G_{n}(z)$ as Lemma 1 for $g_{n}(z)$. Thus it yields the same conclusion, namely that $\Delta_{a}(z)$ is $\Gamma, \rho>1$, which is the first part of $(b)$ of Theorem 3.

Consider

$$
\tau^{\prime}=\lambda z e^{-z} \text {. }
$$

For $\rho=e / \lambda \leqq 1$, the level curve $\left|\tau^{\prime}\right|=1$ consists of two curves:

$$
\left.\begin{array}{l}
\Gamma_{1}=\left\{z|| \lambda z e^{-z}|=1,| z \mid \leqq 1\right\}, \\
\Gamma_{1}^{\prime}=\left\{z !\left|\lambda z e^{-z}\right|=1,|z| \geqq 1\right\} .
\end{array}\right\}
$$


Denote the interior of $\Gamma_{1}$ by $I^{\prime}$, the domain left of $\Gamma_{1}^{\prime}$ by $\mathrm{II}^{\prime}$, and the domain bounded by $\Gamma_{1}$ and $\Gamma_{1}^{\prime}$ by III'. Note that $I^{\prime} \subseteq I, I^{\prime} \subseteq$ II, III' $\subseteq$ III. We shall prove the following lemma.

LEMMA 3. For $\rho=e / \lambda \leqq 1, z \neq 1$ we have

(a) $G_{n}(z)=a\left(\lambda z e^{-z}\right)^{n}\left(1+\eta_{n}(z)\right)$,

for $z$ in I, III or on $\Gamma$.

(b) $G_{n}(z)=1+a\left(\lambda z e^{-z}\right)^{n}\left(1+\eta_{n}^{\prime}(z)\right)$,

for $z$ in II, III or on $\Gamma^{\prime}$. and 2 .

$\eta_{n}(z)$ and $\eta_{n}^{\prime}(z)$ have the same limit behavior as the corresponding functions in Lemmas 1

The above lemma is a direct consequence of Lemma 1. For instance, to prove part $(a)$, one can use (7) and part (a) of Lemma 1 to get

$$
\begin{aligned}
G_{n}(z) & =a e^{-n z} \lambda^{n} z^{n}+(1 / \sqrt{ }(2 \pi n))\left(z e^{-z}\right)^{n}(z /(1-z))\left(1+\varepsilon_{n}(z)\right) \\
& =a\left(\lambda z e^{-z}\right)^{n}\left[1+(e / \lambda)^{n}(1 / a \sqrt{ }(2 \pi n))(z /(1-z))\right]\left(1+\varepsilon_{n}(z)\right) .
\end{aligned}
$$

Since $e / \lambda \leqq 1$, the expression in the square brackets will approach 1 uniformly. Thus part (a) is proved.

From Lemma 3, we have

$$
\lim G_{n}(z)=\left\{\begin{array}{l}
0 \text { for } z \text { in } I^{\prime} \\
1 \text { for } z \text { in } I^{\prime} \\
\infty \text { for } z \text { in } I^{\prime}
\end{array}\right.
$$

uniformly in every region which is entirely located in $\mathrm{I}^{\prime}$, II' and $\mathrm{III}^{\prime}$, respectively. Consequently, for large $n, G_{n}(z) \neq 1$ in $\mathrm{I}^{\prime}$ or in III'. As for $z$ in $\mathrm{II}^{\prime}$ and $\Gamma_{1}^{\prime}$, part $(b)$ of Lemma 3 shows that $\lim \left(G_{n}(z)-1\right) / a\left(\lambda z e^{-z}\right)^{n}=1$. Thus for $n$ sufficiently large, a theorem due to Hurwitz yields that $G_{n}(z)-1 \neq 0$ in $\mathrm{II}^{\prime}, \mathrm{III}^{\prime}$ or on $\Gamma_{1}^{\prime}$. The only possible location of $\Delta_{a}(z)$ then is $\Gamma_{1}$. However, that $\Delta_{a}(z)$ occupies every point of $\Gamma_{1}$ is a consequence of Theorem 4 below. This completes the second part of part $(b)$ of Theorem 3.

4. An asymptotic distribution of the zeros and the $a$-points of $f_{n}(\zeta)$. Using Lemma 1 , Szegö not only proved that the derived set of the zeros of $s_{n}(z)$ and $s_{n}(z)-a$ is identical to $\Gamma$, but also that its elements are positioned along any arc of $\Gamma$ in such a way that the distribution along the arc is asymptotically equal to the change in $(1 / 2 \pi)\left(\arg \left(z e^{1-z}\right)^{n}\right)$ along the arc. We shall call such a distribution uniform. Obviously the distribution of the zeros of $f_{n}(\zeta)$ along $\Gamma$ in the $z$-plane is uniform. Also, since Lemma 2 is the same as Lemma 1, the distribution of the $a$-points of $f_{n}(\zeta)$ along $\Gamma$, when $\rho>1$, is uniform. As for the distribution of the $a$-points of $f_{n}(\zeta)$ for $\rho \leqq 1$, we shall show that it is uniform along $\Gamma_{1}$ in the $z$-plane.

Let $0<r<1<R, 0<\theta_{1}<\theta_{2}<2 \pi$. Consider the region in the $\tau^{\prime}$-plane bounded by 
two line segments and two circular arcs whose vertices are $r e^{i \theta_{1}}, R e^{i \theta_{1}}, R e^{i \theta_{2}}$, and $r e^{i \theta_{2}}$. Let $D$ be the region in the $z$-plane whose image in the $\tau^{\prime}$-plane under (8) is the above region.

Theorem 4. Let $r, R, \theta_{1}, \theta_{2}$ be chosen as before. For sufficiently large $n$, let $N\left(r, R, \theta_{1}, \theta_{2}\right)$ be the number of zeros of $G_{n}(z)-1$ in $D$ when $\rho \leqq 1$. Then

$$
N\left(r, R, \theta_{1}, \theta_{2}\right)=n\left(\theta_{2}-\theta_{1}\right) / 2 \pi+O(1) .
$$

Proof. For every $n$, we associate with $D$ two regions $D_{n}^{-}, D_{n}^{+}$, such that $D_{n}^{-} \subset D \subset D_{n}^{+}$. In order to construct $D_{n}^{-}$, for example, replace the right-hand boundary of $D$ by another curve whose image under (8) consists of two line segments and one circular arc connecting the following points in the positive direction: $r e^{i\left(\theta_{1}+\beta / n\right)}, \frac{1}{2}(1+R) e^{i\left(\theta_{1}+\beta / n\right)}, \frac{1}{2}(1+R) e^{i \theta_{1}}, R e^{i \theta_{1}}$, and such that

$$
n \theta_{1}+\beta \equiv-\alpha+\pi(\bmod 2 \pi) \text {, where } \alpha=\arg a \text { and } 0 \leqq \beta<2 \pi .
$$

Replace the left-hand boundary of $D$ by a similar interior curve. Thus $D_{n}^{-} \subset D$. The replacement of the right and left boundary parts of $D$ by two exterior curves constructed in a way similar to the above is $D_{n}^{+}$. Thus $D \subset D_{n}^{+}$. Let $N_{n}^{-}, N_{n}^{+}$be the number of zeros of $G_{n}(z)-1$ in $D_{n}^{-}, D_{n}^{+}$, respectively. We shall show that

and

$$
N_{n}^{-}=n\left(\theta_{2}-\theta_{1}\right) / 2 \pi+O(1)
$$

$$
N_{n}^{+}=n\left(\theta_{2}-\theta_{1}\right) / 2 \pi+O(1) \text {. }
$$

Since $N_{n}^{-} \leqq N_{n} \leqq N_{n}^{+}$, Theorem 4 will then be proved. We shall show the above for $D_{n}^{-} ; D_{n}^{+}$is handled similarly.

Let $A, B, C, D, E, F, G, H$, be the points on the boundary of $D_{n}^{-}$which are the images under (8) of the points $\left.r e^{i\left(\theta_{2}-\beta / n\right)}, r e^{i\left(\theta_{1}+\beta / n\right.}\right), \frac{1}{2}(1+R) e^{i\left(\theta_{1}+\beta / n\right)}, \frac{1}{2}(1+R) e^{i \theta_{1}}, R e^{i \theta_{1}}, R e^{i \theta_{2}}$, $\frac{1}{2}(1+R) e^{i \theta_{2}}, \frac{1}{2}(1+R) e^{i\left(\theta_{2}-\beta / n\right)}$, respectively. Let

$$
F(z)=a\left(\lambda z e^{-z}\right)^{n} \text {. }
$$

In what follows $n$ is chosen large enough to satisfy the different statements mentioned below. From (8) and (11) it follows that

for $z$ on $A B$, while

$$
|(F(z)-1) / F(z)|>1 /|a| r^{n}-1>\frac{1}{2}
$$

$$
|(F(z)-\vdots) / F(z)|>1-1 /|a| R^{n}>\frac{1}{2}
$$

for $z$ on $E F$. Since the curve $B C$ is mapped by (11) onto a line segment joining $-|a| r^{n}$ and $-|a|((1+R) / 2)^{n}, F(z)$ is closer to the origin than to $(1,0)$ when $z$ traverses $B C$. Hence $|(F(z)-1) / F(z)|>1$ for $z$ on $B C$. For $z$ on $C D$ or $D E,|(F(z)-1) / F(z)|>1-2^{n} /|a|(1+R)^{n}>\frac{1}{2}$. In short, $|(F(z)-1)| F(z) \mid>\frac{1}{2}$ whenever $z$ is on the curve $A B C D E F$. Similarly, the above inequality holds on the rest of the boundary of $D_{n}^{-}$. Thus

$$
|F(z)-1|>\frac{1}{2}|F(z)|
$$

for $z$ on the boundary of $D_{n}^{-}$and for sufficiently large $n$. 
From Lemma 3, part (a), one obtains

$$
G_{n}(z)-1=F(z)-1+F(z) \eta_{n}(z) \text {. }
$$

Since $\eta_{n}(z) \rightarrow 0,\left|\eta_{n}(z)\right|<\frac{1}{2}$ for $z$ on the boundary of $D_{n}^{-}$. From this and (12) it follows that

$$
|F(z)-1|>\frac{1}{2}|F(z)|>\left|F(z) \eta_{n}(z)\right|,
$$

for $z$ on the boundary of $D_{n}^{-}$. Rouche's theorem yields that $F(z)-1$ and $F(z)-1+F(z) \eta_{n}(z)$ have the same number of zeros in $D_{n}^{-}$. It follows from (13) that the number of zeros of $G_{n}(z)-1$ is the same as the number of zeros of $a\left(\lambda z e^{-z}\right)^{n}-1$ in $D_{n}^{-}$. Note that the change of the argument of $a\left(\lambda z e^{-z}\right)^{n}-1$ as $z$ traverses the boundary of $D_{n}^{-}$is determined by the change of the argument as $z$ traverses the arc $E F$ except for an additive term which remains bounded for sufficiently large $n$. Using the argument principle, we get

$$
N_{n}^{-}=n\left(\theta_{2}-\theta_{1}\right) / 2 \pi+O(1) \text {. }
$$

Similarly $N_{n}^{+}=n\left(\theta_{2}-\theta_{1}\right) / 2 \pi+O(1)$ and (10) follows.

\section{REFERENCES}

1. H. S. Al-Amiri, The a-points of Faber polynomials, Bull. College Sci. Baghdad 8 (1965), 1-25.

2. A. Hurwitz and R. Courant, Funktionentheorie (Interscience Publishers, 1925). $50-64$.

3. G. Szegö, Uber eine Eigenschaft der Exponentialreihe, Sitzungsber. Ber. Math. Ges. 23 (1924),

4. J. Ullman, Studies in Faber polynomials, Trans. Amer. Math. Soc. 94 (1960), 515-528.

Bowling Green State University

OHIO, U.S.A. 